\title{
Características clínico-epidemiológicas y evolución del tratamiento en pacientes con úlceras corneales
}

\author{
*Jenny Ku Lozano ${ }^{1}$, Margarita Samudio ${ }^{2}$, Jason A. Penniecook-Sawyers ${ }^{1}$, Sonia \\ Abente $^{2}$, Carolina Duré ${ }^{2}$ \\ ${ }^{1}$ Fundación Visión, Servicio de Oftalmología. Asunción, Paraguay \\ ${ }^{2}$ Universidad Nacional de Asunción, Instituto de Investigaciones en Ciencias de la Salud. \\ Paraguay
}

\author{
Cómo citar este artículo/ \\ How to reference this article:
}

\begin{abstract}
Ku Lozano JK, Samudio M, PenniecookSawyers J, Abente $S$, Duré C. Características clínico-epidemiológicas y evolución del tratamiento en pacientes con úlceras corneales. Mem. Inst. Investig. Cienc. Salud. 2019; 17(1): 16-24
\end{abstract}

\begin{abstract}
R E S U M E N
Las úlceras corneales de origen infeccioso son una emergencia oftalmológica que amenaza la visión y la integridad estructural del ojo, causando ceguera en 1,5 a 2 millones de casos al año por lo que requiere tratamiento inmediato. El objetivo fue determinar el patrón epidemiológico, factores de riesgo y efectividad del tratamiento en pacientes con úlcera corneal en la Fundación Visión en el periodo 2015-2017. Estudio de cohorte prospectivo donde se evaluó 53 pacientes con úlcera corneal de causa infecciosa que consultaron en la Fundación Visión en el periodo 2015-2017, y tuvieron un seguimiento mínimo de 4 meses. Se encontró un predominio del sexo masculino $(69,8 \%)$, edad $45-65$ años $(49,1 \%)$, casados $(54,7 \%)$, de condición socioeconómica baja $(56,6 \%)$, agricultores $(26,4 \%)$, con antecedente de traumatismo corneal $(69,8 \%)$ o uso de lentes de contacto $(17 \%)$. El agente etiológico fue bacteriano en el $49,1 \%$ y micótico en el 37,7\%; para los de origen bacteriano el tratamiento más empleado fueron colirios fortificados de Cefazolina + Gentamicina y Natamicina/Fluconazol en caso de ser micótico. La evolución fue favorable en 90,9\% y $80,0 \%$ de los bacterianos y micóticos, respectivamente. Los grupos de riesgo detectados fueron pacientes varones, agricultores, de condición socioeconómica baja, con antecedente de traumatismo corneal o uso de lentes de contacto. La efectividad del tratamiento utilizado fue mayor al $80 \%$, siendo en su mayoría patógenos bacterianos.
\end{abstract}

Palabras clave: úlcera corneal, queratitis, factores de riesgo, tratamiento, Fundación Visión, Paraguay.

\section{Clinical-epidemiological characteristics and treatment evolution of patients with corneal ulcer}

\section{A B S T R A C T}

Infectious corneal ulcers are an ophtalmological emergency that threatens sight and eyes integrity, causing blindness in 1.5-2 million of people per year. The objective was to determinate epidemiological pattern, risk factors and treatment efficiency in patients with corneal ulcer in Fundacion Vision from 2015 to 2017. This was a prospective cohort study, 53 patients with infectious corneal ulcer were examined in Fundacion Vision from 2015 to 2017 , and were followed for at least 4 months. Predominance of males $(69.8 \%)$, aged $45-$ 65 years $(49.1 \%)$, married $(54.7 \%)$, low socio-economic status $(56.6 \%)$, farmers $(26.4 \%)$, with a history of corneal trauma $(69.8 \%)$ or contact lenses users $(17 \%)$ were found. The etiological agent was bacterial in $49.1 \%$ and mycotic in $37.7 \%$; the most accurate 
treatment for those of bacterial origin was fortified eye drops of Cefazolin+Gentamicin and Natamycin/Fluconazole in case of being fungal. The evolution was favorable in $90.9 \%$ and $80.0 \%$ of the bacterial and mycotic infections, respectively. High risk groups detected were male patients, farmers, low socio-economic status, with a history of corneal trauma or contact lenses user. On the other hand, the effectiveness of the treatment was greater than $80 \%$, and the infections were mostly caused by bacterial pathogens.

Keywords: corneal ulcer, keratitis, risk factors, treatment, Fundación Vision, Paraguay.

\section{INTRODUCCION}

A nivel mundial, la incidencia anual de ceguera, definida por la OMS (Organización Mundial de la Salud) como una agudeza visual inferior a 3/60 (20/400), o una pérdida del campo visual a menos de $10^{\circ}$ en el mejor ojo ${ }^{(1)}$ causada por úlcera debido a queratitis infecciosa o traumática es de 1,5 a 2 millones de casos, constituyendo motivo de interés y preocupación para los oftalmólogos por sus potenciales consecuencias.

Se estima que ocurren alrededor de 30000 casos de queratitis infecciosa en los EE.UU anualmente ${ }^{(2)} \mathrm{y}$, aproximadamente 20 individuos por cada 10000 de ellos, son usuarios de lentes de contacto $^{(3)}$. Mientras que en Gran Bretaña indican 4000 casos anuales de queratitis infecciosa que requieren hospitalización ${ }^{(4)}$. Todo ello se resume a un costo estimado de visitas a los oftalmólogos de $\$ 175$ millones y 250000 horas oftalmólogo/clínica anuales ${ }^{(5)}$.

En América Latina se han reportado pocos estudios aún, y dado que es una importante causa de ceguera irreversible pero prevenible en los adultos alrededor del mundo es vital su estudio $^{(1,2)}$.

Las úlceras corneales causadas por queratitis infecciosa, son el resultado de una alteración en la barrera de protección natural, con afectación no sólo de la capa más superficial (epitelio), sino también más profundas, cuya consecuencia es una cicatriz que compromete la visión y por consiguiente genera un impacto socioeconómico importante ${ }^{(5,6)}$. Cabe resaltar que también existen patógenos que pueden producir úlceras corneales aún con la barrera epitelial intacta, como son Neisseria meningitidis, Neisseria gonorrhoeae, Corynebacterium diphteriae, Haemophilus aegyptius y Listeria monocytogenes ${ }^{(7)}$.

Se han mencionado numerosos factores de riesgo, siendo el principal el uso de lentes de contacto (36-56\%), luego, traumatismos oculares no quirúrgicos: como abrasión corneal y cuerpos extraños (20-25\%), Ojo seco (15\%), alteraciones palpebrales como triquiasis, lagoftalmos, ectropion, entropión, exoftalmos (6\%), cirugía intraocular (3\%) y finalmente el uso de esteroides tópicos es un factor coadyuvante, así como condiciones sistémicas (malnutrición, diabetes mellitus, alcoholismo, tabaquismo) y el uso de agentes inmunosupresores que condicionen a la sequedad ocular ${ }^{(8,9)}$.

Cuando se desarrolla una úlcera corneal, suele aparecer quemosis e inyección conjuntival, edema palpebral y perilesional, disminución de la visión, intenso dolor ocular, lagrimeo, fotofobia, secreción purulenta y sensación de cuerpo extraño y dependiendo de la gravedad puede existir adelgazamiento estromal, endotelial, hipopión e incluso perforación ocular $^{(8)}$.

La situación en nuestro medio es muy compleja ya que la mayoría de los pacientes consultan tardíamente, con tratamiento previo instaurado, frecuentemente inadecuado. Esto produce alteraciones en las características del proceso, llegando incluso a enmascarar al verdadero agente etiológico. Es por ello que debemos ser muy acuciosos al momento de realizar la anamnesis del paciente, así como valorar su estado físico, exámenes auxiliares laboratoriales, siendo un punto crucial la comunicación estrecha con el microbiólogo ${ }^{(7-10)}$.

En la Fundación Visión - Asunción no se han realizado trabajos que permitan conocer la situación actual de las úlceras por queratitis infecciosas por lo que el propósito del presente estudio es lograr un análisis de las características epidemiológicas, establecer un diagnóstico clínico-microbiológico de las úlceras corneales por queratitis infecciosa e indicar los resultados de los distintos métodos de tratamiento utilizados.

\section{METODOLOGIA}

Se realizó un estudio observacional analítico, y de cohorte prospectivo en la Clínica Central de la Fundación Visión, Asunción - Paraguay, institución dedicada a la atención oftalmológica de la población paraguaya, cuyo número de pacientes atendidos durante el periodo de estudio (2015 al 2017) fue de 167.395 personas. 
Se incluyó todo paciente con queratitis que presentó úlcera corneal $>1,5 \mathrm{~mm}$ independientemente de su ubicación (central o periférica), de causa infecciosa (considerándose como bacteriana o micótica de acuerdo al resultado del análisis laboratorial), cuyos datos sociodemográficos, clínicos y microbiológicos fueron registrados en la ficha de admisión de la Fundación Visión tras sus atenciones oftalmológicas durante el periodo 2015 - 2017, con un seguimiento mínimo de 4 meses. Se excluyeron pacientes que presentaron úlcera corneal de causa no infecciosa o cuyas fichas de admisión se encontraron incompletas y no se les realizó cultivo microbiológico.

\section{Técnicas y Procedimientos de recolección de información}

Se realizó la evaluación y el llenado de ficha de datos de los pacientes con úlcera corneal por queratitis infecciosa que consultaron en la clínica central de la Fundación Visión durante el periodo 2015- 2017 y que cumplieron con los criterios de inclusión y de exclusión. En base a una ficha de recolección de datos, que contenía información sobre las características demográficas (edad, sexo, etnia, estado civil condición socioeconómica, ocupación laboral Ubicación geográfica), características clínicas de los pacientes (dolor, ojo rojo, fotofobia hipopion), además de la agudeza visual antes y después del tratamiento.

Se valoró la agudeza visual en el ojo afectado inicial y post-tratamiento antibiótico, con la mejor corrección posible mediante el uso del test de Snellen a 6 metros de distancia del paciente, que cuantificó de manera subjetiva la visión del paciente, desde el rango 20/20 hasta 20/200.

Características de la úlcera: el tamaño se clasificó dentro de los siguientes rangos: pequeña: <2 mm, mediana: 2 - $5 \mathrm{~mm}$ y grande: $>5 \mathrm{~mm}$; la localización en central o periférica.

Los factores de riesgo fueron clasificados en: sistémicos (HTA, DM) y locales (traumatismos oculares, uso de lentes de contacto; uso crónico de medicamentos tópicos).

El tratamiento fue indicado de acuerdo a cinco esquemas. El tratamiento para úlcera bacteriana incluía dos esquemas: Esquema 1: Cefazolina + Gentamicina, una gota horaria de cada uno y Esquema 3: Vancomicina + Ceftazidima, una gota horaria de cada uno.

El tratamiento para úlcera micótica incluía un esquema: Esquema 5: Natamicina y/o Fluconazol, una gota horaria de cada uno.

El tratamiento para úlcera micótica y bacteriana dos esquemas: Esquema 2: Cefazolina + Gentamicina+Natamicina/Fluconazol, una gota horaria de cada uno y Esquema 4: Vancomicina +Ceftazidima+Natamicina/Fluconazol, una gota horaria de cada uno

Respuesta al tratamiento: Se valoró la respuesta al tratamiento como favorable si presentó la resolución completa del cuadro o al menos la ausencia de 3 de los signos y síntomas: dolor, ojo rojo, fotofobia o disminución de la agudeza visual (como máximo 2 líneas por debajo de la agudeza visual pre tratamiento) valorado por la cartilla de Snellen como mínimo a los 4 meses. Se definió como respuesta desfavorable si existía la persistencia de los signos y síntomas tras el tratamiento indicado, donde se valoró la necesidad de un cambio de esquema o una cirugía.

\section{Gestión y análisis de los datos}

El cálculo del tamaño de la muestra fue basado en una efectividad del tratamiento de $90 \%$. Por lo tanto para un nivel de confianza del $95 \%$, una proporción esperada de 0,90 y un error de $9 \%$; el tamaño mínimo a reclutarse era de 49 pacientes.

Los datos fueron consignados en una plantilla electrónica de Microsoft Excel 2017 y posteriormente analizados por EpiInfo (CDC, Atlanta). Los resultados se presentan en tablas de frecuencias absolutas y porcentuales.

\section{Asuntos Éticos}

Los datos personales de los participantes de este estudio se mantuvieron en forma confidencial y fueron usados sólo con fines científicos, respetando de esta manera los principios éticos de no maleficencia y justicia con fines productivos de acuerdo al código de Nuremberg y la Declaración de Helsinski.

\section{RESULTADOS}

En el periodo de estudio (2015 al 2017), 53 pacientes cumplieron con los criterios de inclusión, encontrándose una mayor frecuencia de pacientes de sexo masculino $(69,8 \%)$, casados $(54,7 \%)$, caucásicos $(60,4 \%)$, de condición socioeconómica baja $(56,6 \%)$, agricultores $(26,4 \%)$, provenientes desde zonas aledañas como el departamento Central $(49,1 \%)$, las edades oscilaban mayormente entre 25 y 65 años. Tabla 1 
Tabla 1. Características demográficas de los pacientes con úlceras corneales infecciosas. $n=53$

\begin{tabular}{|c|c|c|c|}
\hline \multicolumn{2}{|c|}{ Características demográficas } & \multirow{2}{*}{$\begin{array}{l}\text { Frecuencia } \\
6\end{array}$} & \multirow{2}{*}{$\begin{array}{c}\text { Porcentaje } \\
11,3\end{array}$} \\
\hline Edad (años) & $<25$ & & \\
\hline & $25-45$ & 14 & 26,4 \\
\hline & $45-65$ & 26 & 49,1 \\
\hline & $>65$ & 7 & 13,2 \\
\hline \multirow[t]{2}{*}{ Sexo } & Masculino & 37 & 69,8 \\
\hline & Femenino & 16 & 30,2 \\
\hline \multirow[t]{2}{*}{ Estado civil } & Casado & 29 & 54,7 \\
\hline & Soltero & 24 & 45,3 \\
\hline \multirow[t]{2}{*}{ Etnia } & Caucásico & 32 & 60,4 \\
\hline & Indígena & 21 & 39,6 \\
\hline \multirow[t]{2}{*}{ Condición socioeconómica } & Baja & 30 & 56,6 \\
\hline & Media & 23 & 43,4 \\
\hline \multirow[t]{9}{*}{ Ocupación } & Agricultor & 14 & 26,4 \\
\hline & Ama de casa & 10 & 18,9 \\
\hline & Comerciante & 5 & 9,4 \\
\hline & Jardinero & 5 & 9,4 \\
\hline & Albañil & 4 & 7,5 \\
\hline & Carpintero & 4 & 7,5 \\
\hline & Estudiante & 4 & 7,5 \\
\hline & Mecánico & 3 & 5,7 \\
\hline & Otras* & 4 & 7,6 \\
\hline \multirow[t]{10}{*}{ Procedencia } & Central & 26 & 49,1 \\
\hline & Canindeyú & 5 & 9,4 \\
\hline & Cordillera & 4 & 7,5 \\
\hline & Caaguazú & 4 & 7,5 \\
\hline & Paraguarí & 4 & 7,5 \\
\hline & San Pedro & 3 & 5,7 \\
\hline & Boquerón & 3 & 5,7 \\
\hline & Concepción & 2 & 3,8 \\
\hline & Caazapá & 1 & 1,9 \\
\hline & Ñeembucú & 1 & 1,9 \\
\hline
\end{tabular}

*Otras: abogado, costurera, médica, repartidor

Dentro de los factores de riesgo locales de las úlceras corneales se encontró como principal: los traumatismos corneales $(69,8 \%)$. También una importante proporción de pacientes era usuario de lentes de contacto (17\%). Entre los sistémicos, un $11 \%$ era diabético. Tabla 2

Tabla 2. Factores de riesgo asociados a úlceras corneales infecciosas

\begin{tabular}{llcc}
\hline & Factores de riesgo & Frecuencia & Porcentaje \\
\hline Local & Traumatismos corneales & 37 & 69,8 \\
& Uso de lentes de contacto & 9 & 17,0 \\
\multirow{2}{*}{ Sistémico } & Diabetes Mellitus & 11 & 20,8 \\
& Hipertensión Arterial & 7 & 13,2 \\
\hline
\end{tabular}

La ubicación de las úlceras corneales infecciosas fue mayormente central (66\%), el tamaño promedio fue de $2-5 \mathrm{~mm}(58,5 \%)$ que corresponde a una úlcera de tamaño mediano y el ojo derecho fue el más afectado $(56,6 \%)$. Se encontró que los agentes causales predominantes fueron las bacterias $(49,1 \%)$ seguido de hongos $(37,7 \%)$, un solo caso de parásito (Acanthamoeba sp.) fue identificado. Tabla 3 
Tabla 3. Características y resultados etiológicos de las úlceras corneales infecciosas

\begin{tabular}{llcc}
\hline \multicolumn{2}{c}{ Características de las úlceras corneales infecciosas } & Frecuencia & Porcentaje \\
\hline Ubicación de las úlceras & Central & 35 & 66,0 \\
& Periférica & 18 & 34,0 \\
Tamaño de las úlceras & Pequeño & 9 & 17,0 \\
& Mediano & 31 & 58,5 \\
& Grande & 13 & 24,5 \\
\multirow{2}{*}{ Ojo afectado } & & & \\
& Derecho & 30 & 56,6 \\
Resultado microbiológico & 23 & 43,4 \\
& Izquierdo & 28 & 49,1 \\
& & 20 & 37,7 \\
& Bacteria & 1 & 1,9 \\
& Hongo & 4 & 7,5 \\
\hline
\end{tabular}

Entre las bacterias, las más frecuentes fueron: Estafilococos coagulasa negativa $(24,5 \%)$ y Pseudomonas aeruginosa (7,5\%); mientras que, entre los hongos, Fusarium sp. fue el más frecuente $(15,1 \%)$. Tabla 4

Tabla 4. Resultados microbiológicos de las úlceras corneales infecciosas

\begin{tabular}{lcc}
\hline Microorganismos & Frecuencia & Porcentaje \\
\hline Bacteria & & \\
Estafilococos coagulasa negativa & 13 & 24,5 \\
Pseudomonas aeruginosa & 4 & 7,5 \\
Staphylococcus aureus & 3 & 5,7 \\
Streptococcus pneumoniae & 3 & 5,7 \\
Klebsiella pneumoniae & 2 & 3,8 \\
Propionibacterium acnes & 1 & 1,9 \\
Propionibacterium sp. & 1 & 1,9 \\
Serratia marcescens & 1 & 1,9 \\
Hongo & & \\
Fusarium sp. & 8 & 15,1 \\
Hongo (sin identificar) & 5 & 9,4 \\
Hongos dematiáceos & 3 & 5,7 \\
Curvularia sp. & 2 & 3,8 \\
Aspergillus sp. & 1 & 1,9 \\
Candida sp. & 1 & 1,9 \\
Exserohilum sp. & 1 & 1,9 \\
Scedosporium sp. & 1 & \\
\hline
\end{tabular}

El tratamiento fue de acuerdo a 5 esquemas, utilizándose mayormente los esquemas 2 y 5 para úlceras infecciosas. La evolución de las úlceras fue mayormente favorable con respecto a las de tipo bacteriano $(92,9 \%)$ comparado al micótico $(80,0 \%)$; y en $100 \%$ de las úlceras de tamaño pequeño y mediano, mientras que en $53,8 \%$ de las úlceras de tamaño grande, que fue significativamente menor que los tamaño mediano y pequeño. Tabla 5 
Tabla 5. Factores asociados a la evolución a los 4 meses de las úlceras corneales infecciosas

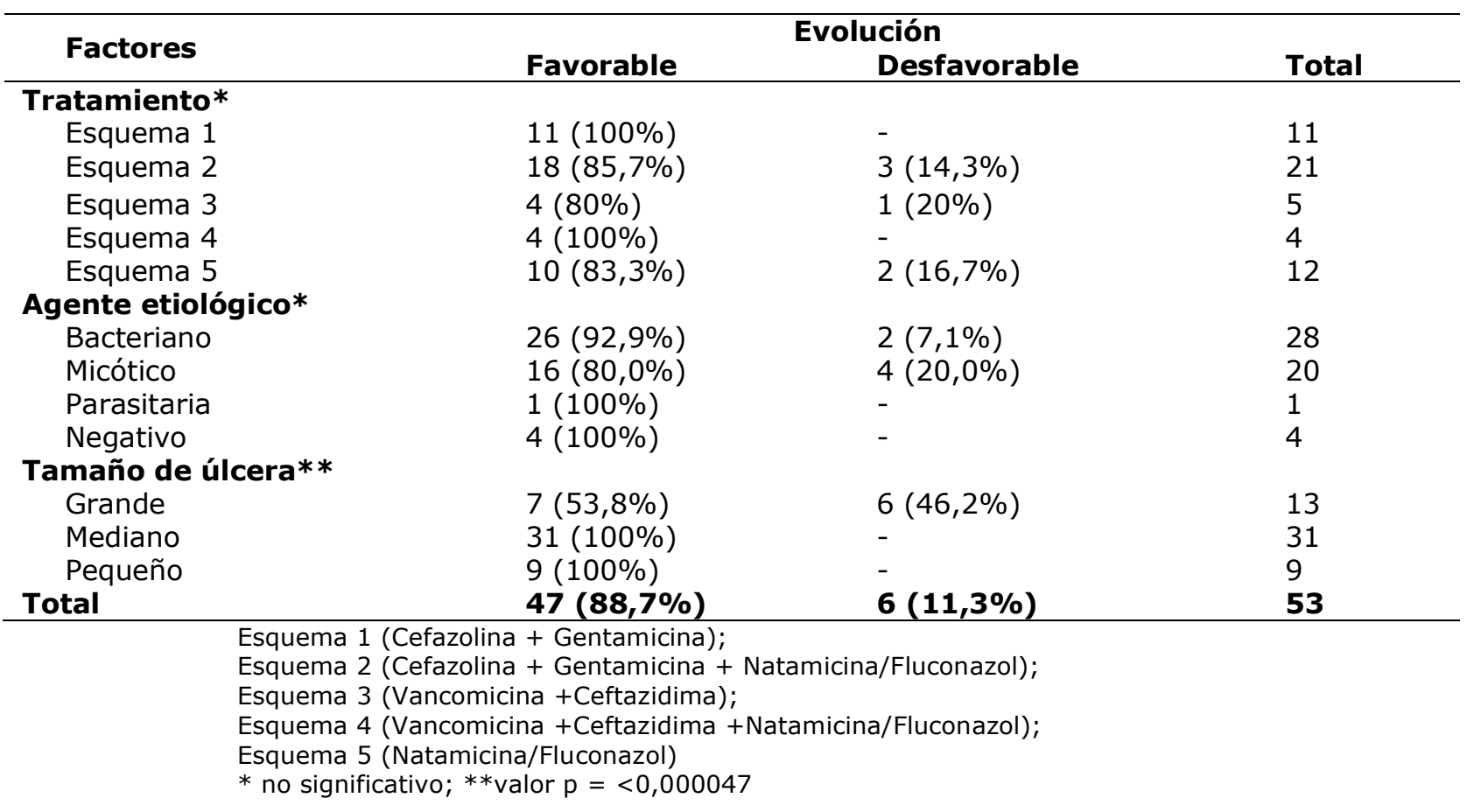

En cuanto a la agudeza visual inicial (previa al tratamiento) comparada con la final (4 meses post tratamiento), se observó una mejoría de 1 a 5 líneas en la cartilla de Snellen en promedio de la mayoría de los pacientes. No obstante, aquellos cuya agudeza visual previa fue no percepción de luz o no valorable, obtuvieron un resultado estacionario, como se puede observar en el cuadro descrito a continuación. Tabla 6

Tabla 6. Agudeza visual previa y posterior al tratamiento de 4 meses de evolución de úlceras corneales infecciosas

\begin{tabular}{|c|c|c|c|c|c|c|c|c|c|c|c|c|}
\hline \multirow{2}{*}{$\begin{array}{l}\text { Pre- } \\
\text { tratamiento }\end{array}$} & \multicolumn{12}{|c|}{ Post - tratamiento } \\
\hline & $20 / 20$ & $20 / 25$ & $20 / 30$ & $20 / 40$ & $20 / 50$ & $20 / 70$ & $20 / 100$ & $\mathrm{CD}$ a $1 \mathrm{~m}$ & MM & $\mathrm{PL}$ & NPL & Total \\
\hline $20 / 30$ & 1 & - & - & - & - & - & - & - & - & - & - & 1 \\
\hline $20 / 40$ & 1 & - & - & - & - & - & - & - & - & - & - & 1 \\
\hline $20 / 50$ & 1 & - & 1 & - & - & - & - & - & - & - & - & 2 \\
\hline $20 / 70$ & 1 & - & 2 & - & - & - & - & - & - & - & - & 3 \\
\hline $20 / 100$ & - & 1 & 2 & - & - & - & - & - & - & - & - & 3 \\
\hline $20 / 200$ & - & - & - & - & 2 & 1 & - & - & - & 1 & - & 4 \\
\hline CD a $1 \mathrm{~m}$ & - & - & 1 & 5 & 1 & 1 & 1 & - & - & - & - & 9 \\
\hline$C D$ a $50 \mathrm{~cm}$ & 1 & 2 & 1 & 1 & 1 & - & - & 1 & - & - & - & 7 \\
\hline MM & - & - & 1 & - & 4 & 2 & - & 1 & 1 & - & 3 & 12 \\
\hline PL & - & - & - & - & 1 & 2 & - & 1 & 1 & - & - & 5 \\
\hline NPL & - & - & - & - & - & - & - & - & - & - & 4 & 4 \\
\hline NV & - & - & - & - & - & 1 & - & - & & - & 1 & 2 \\
\hline Total & 5 & 3 & 8 & 6 & 9 & 7 & 1 & 3 & 2 & 1 & 8 & 53 \\
\hline
\end{tabular}

Cuenta dedos (CD); Movimiento de Manos (MM); Percibe Luz (PL); No percibe Luz (NPL); No valorable (NV)

\section{DISCUSION}

En esta investigación se evaluaron 53 pacientes con úlceras corneales infecciosas durante un periodo de 3 años (2015 al 2017), encontrándose un predominio del sexo masculino sobre el femenino, proporción varón: mujer $2: 1$, lo cual concuerda con los diversos trabajos realizados en India (1.6:1), China (1.5:1), Brasil (6.4:1) y Paraguay $(3: 1)^{(12-15)}$. Ello se debe probablemente a que los varones, al tener actividades ocupacionales más arriesgadas y al no usar la protección necesaria, tengan mayor riesgo ${ }^{(9)}$. Sin embargo, en países donde el uso de lentes de contacto es el factor de riesgo prevalente, se encuentran un predominio de pacientes del sexo femenino ${ }^{(9,10)}$. Además, se encontró que las personas entre 45 - 65 años de edad $(49,1 \%)$, casados $(54,7 \%)$ y con condición 
socioeconómica baja $(56,6 \%)$ fueron el grupo más afectado, lo que coincide con lo encontrado por Satpathi et al en India ${ }^{(10)}$. Esto puede deberse a que a partir de los 25 años se establece a la población económicamente activa como base de la sociedad y pilar laboral de su familia. Así también, cabe considerar que a los 40 años, la presbicia cumple un rol primordial en el proceso de enfoque de objetos ubicados a una distancia cercana/media, cuya incidencia aumenta proporcionalmente respecto a la edad, lo cual se puede considerar como un factor interviniente.

En cuanto a los factores predisponentes de úlcera corneal por queratitis infecciosa se encontró que se presentó mayormente en los pacientes que tuvieron un traumatismo corneal $(69,8 \%)$ y los usuarios de lentes de contacto $(17 \%)$. esto se encuentra invertido en los países desarrollados, donde el uso de lentes de contacto es más accesible y común. La incidencia mundial de uso de lentes de contacto es de 3 a 5 por 10000 usuarios por año ${ }^{(12)}$, y la incidencia de queratitis infecciosa varía de acuerdo al tipo de lente que se usa, ya que mientras las lentes blandas de uso diario presentan una incidencia de 1.9-4.1 por 10000 personas/año, las lentes blandas de uso extendido tienen una incidencia diez veces mayor(19.5 por 10000 personas/año), lo cual se atribuye a alteraciones en la superficie corneal $^{(3,11)}$.

Por otra parte, independientemente del tipo de lente, tanto un mal hábito de limpiezadesinfección como el uso extendido de los mismos (más de diez horas por día, el uso nocturno o por más de seis días) incrementan sensiblemente el riesgo de infección corneal, un promedio de 6.5 veces $^{(11,12)}$.

Muchos investigadores encontraron que las úlceras infecciosas involucraban a trabajadores de zonas rurales, agricultores, metalúrgicos o carpinteros, quienes se lastimaban con pequeñas esquirlas metálicas, astillas de maderas, fragmentos de otros materiales o por abrasión física o química ${ }^{(6,13-15)}$; lo cual coincide con lo encontrado en nuestro estudio, donde un $26.4 \%$ fueron agricultores. Así también, otro estudio realizado en India concluye que de las úlceras micóticas producidas por trauma ocular $(60.15 \%)$, $52.32 \%$ se presentaron en agricultores ${ }^{(16)}$.

Las características clínicas de las úlceras infecciosas no son patognomónicas. Muchas veces no podemos definir un hallazgo biomicroscópico que nos permita identificar un microorganismo determinado ${ }^{(5,15,16)}$.

La mayoría de las úlceras en este estudio tenían una ubicación central (66\%) y de tamaño mediano $(58,5 \%)$, e invasión de $2 / 3$ de la córnea, lo que implica un pronóstico visual reservado. Estos datos son similares a los obtenidos por los estudios realizados en Paraguay y Malasia, donde se encontró la presencia de úlcera de ubicación central en $38 \%$ y $69 \%$ respectivamente ${ }^{(17,18)}$.

Por otro lado, las úlceras infecciosas fueron en su mayoría producida por bacterias $(49,1 \%)$, seguida de los hongos $(37,7 \%)$, concordando con los hallazgos del estudio efectuado por Al-Gafri por un periodo de 3 años en Muscat, reportándose que $92 \%$ de los casos fueron causados por bacterias y $7 \%$ por hongos, mientras que un $1 \%$ fue causado por ambos $^{(19)}$. Asimismo, en un estudio realizado por 13 años en el Hospital de Clínicas de Paraguay, de 580 pacientes se observó que $54 \%$ eran de origen bacteriano, $45 \%$ micótico, y $1 \%$ micótico y bacteriano ${ }^{(17)}$.

En nuestro estudio, se encontró que los patógenos bacterianos más comunes fueron Estafilococos coagulasa negativa (ECN) (24,5\%), Pseudomonas aeruginosa $(7,5 \%)$ y Staphylococcus aureus y Streptococcus pneumoniae $(5,7 \%)$; mientras que de los patógenos fúngicos, Fusarium sp. fue el más frecuente $(15,1 \%)$, seguido de los hongos dematiáceos $(5,7 \%)$, lo que coincide con los resultados de Laspina et al, quien obtuvo: Estafilococo coagulasa negativa (29\%), Pseudomonas aeruginosa (16\%), Streptococcus pneumoniae $(10,5 \%)$ y Staphylococcus aureus $(8,1 \%)^{(20)}$. Sin embargo, como ya se ha mencionado en anteriores estudios realizados en Paraguay, cabe resaltar que se desconoce el rol exacto de los estafilococos coagulasa negativa como agentes etiológicos, debido a que constituyen microbiota normal del ojo, siendo aislados en medio de enriquecimiento en $90 \%$ de los casos. No obstante, estas bacterias en condiciones apropiadas son potencialmente patógenas para el ojo y constituyen los agentes etiológicos más frecuentes de endoftalmitis $(5,6,8,9,17,20)$.

Varios estudios sobre úlceras infecciosas de la India indican a Aspergillus sp. como el agente causal más frecuente, seguido por Fusarium y Candida ${ }^{(21)}$. En los Estados Unidos son Candida y Aspergillus en la zona norte y Fusarium en la zona sur (22); mientras que, en países latinoamericanos, en especial Brasil y Paraguay; Fusarium es el agente más 
frecuente seguido de $A s p e r g i l l u s^{(20,23)}$. Pese a estos resultados, en nuestro estudio si bien es cierto Fusarium sp. fue el más frecuente $(15,1 \%)$, los hongos dematiáceos $(5,7 \%)$ y Curvularia sp. $(3,8 \%)$ ocuparon el segundo y tercer lugar, respectivamente. Éstos últimos datos coinciden con lo reportado por Singh ${ }^{(21)}$.

Se propone que el manejo inicial de cualquier proceso donde se sospeche de infección microbiana, debe ser con la recolección de muestras para cultivo y tinciones (40-60\%), posterior a lo cual se iniciará terapia antimicrobiana empírica, con el objetivo de detener las alteraciones estructurales, promover la curación del estroma y la reepitelización corneal $^{(3,16,21,22)}$; pero se debe considerar que no todas las bacterias serán sensibles a los antibióticos de amplio espectro, por lo que el tratamiento previo puede no ser efectivo y que, mientras más demore el inicio del tratamiento, el paciente presenta un mayor riesgo de lesiones corneales permanentes secuelares ${ }^{(8,9,14)}$.

Dentro del tratamiento establecido: los colirios fortificados, tenemos: cefazolina ( 50 $\mathrm{mg} / \mathrm{ml}$ ) en combinación con gentamicina $(16 \mathrm{mg} / \mathrm{ml})$, lo que constituye el esquema 1; mientras que su asociación con natamicina $(5 \%)$ /fluconazol $(0,2 \%)$ establece el esquema 2. Además, la combinación de vancomicina $(25 \mathrm{mg} / \mathrm{ml})$ y ceftazidima conforma el esquema 3 ; si se agrega natamicina $(5 \%)$ /fluconazol $(0,2 \%)$ se habla del esquema 4 y finalmente el esquema 5 lo constituye es uso de natamicina/fluconazol ${ }^{(20)}$.

Este estudio utilizó mayormente los esquemas 2 y 5 para úlceras infecciosas. Cabe resaltar que, en el esquema 5, la natamicina es la droga de primera elección por su gran acción contra hongos filamentosos y bajo costo, pero ya que en Paraguay es difícil su obtención, se suele utilizar fluconazol. En nuestra institución se usa una gota del antibiótico elegido cada 30 minutos inicialmente, luego cada hora y así sucesivamente dependiendo de la evolución y respuesta de las defensas del huésped, puede llegar a aplicarse entre 3 a 8 veces al día, como se indicó en este estudio.

Es conocida la efectividad de las drogas usadas en este estudio, pero tienen algunas desventajas inherentes, como son: su vida limitada, mayor toxicidad corneal y la irritación ocular que le ocasionan al paciente. Además, algunos meta-análisis han comparado la acción de las fluoroquinolonas en relación con la combinación cefazolina/aminoglicósido sin encontrar diferencias significativas con respecto al tiempo y éxito de tratamiento o complicaciones; sin embargo, sí se demostró que eran más tolerables, por lo que se indicó su uso como primera línea ${ }^{(10,15,20)}$.

Al haber iniciado el tratamiento antibiótico es muy importante realizarle seguimiento estrecho de la infección corneal, pues el factor más importante es la respuesta clínica al tratamiento, es decir, se valora la sintomatología del paciente, el tamaño de la úlcera y de acuerdo a ello se decide la continuación o variación del tratamiento, para evitar la resistencia (ya sea cambiando el medicamento ó agregando otro).

Sharma (2012) encontró que la exposición repetida de la microbiota ocular a los antibióticos tópicos favorecía la producción de cepas bacterianas multirresistentes (cepas de Staphylococcus aureus y Enterococcus sp.), observándose un aumento de la resistencia de 3-5 antibióticos de uso común (82\%) y $68 \%$ de las cepas de estafilococo coagulasa negativa. Estos reportes alertan sobre el surgimiento de multirresistencia a antibióticos tópicos usados rutinariamente en infecciones oculares en la comunidad, lo que puede generar una mayor tasa de complicaciones oculares y un deterioro en el pronóstico visual. Es por ello que, si el tratamiento médico no funciona, serán necesarias otras opciones $^{(6,15,24)}$. En caso contrario, si se determina que el tratamiento está funcionando (disminuye la sintomatología, el tamaño de la úlcera, entre otros) se debe decidir propiciamente cuándo reducir los medicamentos ${ }^{(24)}$. En nuestro estudio, se obtuvo una evolución mayormente favorable con respecto a las úlceras infecciosas de tipo bacteriano $(92,9 \%)$ comparado al micótico $(80,0 \%)$; y en $100 \%$ de las úlceras de tamaño pequeño y mediano, mientras que en $53,8 \%$ de las úlceras de tamaño grande. Este resultado puede ser debido a la agresiva invasión corneal del agente etiológico y/o falta de adhesión al tratamiento del paciente.

Finalmente, con respecto a la agudeza visual inicial (previa al tratamiento) comparada con la final ( 4 meses post tratamiento), se observó una mejoría de 1 a 5 líneas en la cartilla de Snellen $(58,5 \%)$, lo cual dependía de la naturaleza del patógeno, la localización de la lesión y la opacidad corneal resultante. Esto se relaciona con el resultado obtenido en un estudio realizado en Malasia, donde los pacientes con opacidad corneal central $(88 \%)$, sólo $12 \%$ recuperaron una agudeza visual de $6 / 18$ o mejor $(20 / 60)^{(18)}$. 
En conclusión, los grupos de riesgo detectados fueron pacientes varones, agricultores, de condición socioeconómica baja, con antecedente de traumatismo corneal o uso de lentes de contacto. La mayoría de los agentes patógenos aislados fueron bacterianos. La efectividad del tratamiento utilizado fue buena, superando el $80 \%$.

\section{CONFLICTOS DE INTERÉS}

Los autores declaran no tener conflictos de interés.

\section{REFERENCIAS BIBLIOGRÁFICAS}

1. Organizacion Mundial de la Salud. Salud Ocular Universal: Un plan de acción mundial para el 2014-2019. 2013;25.

2. Gritz D, Kumar A, Holsclaw D, Porco T, Smith S, Whitcher JP, et al. Epidemiology of ulcerative keratitis in Northern California. Arch Ophthalmol 2010;128(8):1022-8.

3. Zimmerman A, Nixon A, Rueff E. Contact lens associated microbial keratitis: practical considerations for the optometrist. Clin Optom. 2016;1.

4. Taube $M$, Del Mar Cendra M, Elsahn A, Christodoulides $M$, Hossain $P$. Pattern recognition receptors in microbial keratitis. Eye. Nature Publishing Group; 2015;29(11):1399-415.

5. Collier SA, Gronostaj MP, Macgurn AK, Cope JR, Awsumb KL. Estimated Burden of Keratitis - United States, 2010. Choice Rev Online. 2013;33(05):33-2731-33-2731.

6. Sharma S. Diagnosis of infectious diseases of the eye. Eye. Nature Publishing Group; 2012;26(2):177-84.

7. Scot K, Hwang F. BMJ Best practices: Keratitis. BMJ Publishing group Ltd 2018; 64 $p$

8. Weisenthal R, Daly M, Freitas D, Feder R. External Disease and Cornea. American Academy of Ophthalmology, 2018-2019; p. 336.

9. Pérez, J. Hervás J. Queratitis infecciosas. Fundamentos, técnicas diagnósticas y tratamiento. Ergon,Allergan;2006.278 p.

10. Satpathi P, Satpathi S. Study of microbial keratitis in central India. J Infect Dev Ctries. 2012;6(3):295-8.

11. Dart J, Edwards K, Naduvilath T, Holden B, Keay L, Stapleton F, et al. Risk Factors for Moderate and Severe Microbial Keratitis in Daily Wear Contact Lens Users. Ophthalmology. Elsevier Inc.; 2012;119(8):1516-21.

12. Lim C, Carnt N, Farook M, Lam J, Tan D, Mehta J, et al. Risk factors for contact lensrelated microbial keratitis in Singapore. Eye. Nature Publishing Group; 2016;30(3):44755.

13. Nicola F. Queratitis infecciosa no viral: Factores predisponentes, agentes etiológicos y diagnóstico de laboratorio. Rev Argent Microbiol. 2005;37(4):229-39.

14. Bety $M$, Ray SL, Daniel $R$, Cecilia MA, Viridiana LC. Md 2017. 2017;9(04):68-76.
15. Ruiz J, Cabrejas L, De Hoz M, Mingo D, Duran S. Características clínicas y microbiológicas en queratitis infecciosas bacterianas en un hospital de tercer nivel. Arch Soc Esp Oftalmol. Sociedad Española de Oftalmología; 2017;92(9):419-25.

16. Deorukhkar S, Katiyar R, Saini $S$. Epidemiological features and laboratory results of bacterial and fungal keratitis: A five-year study at a rural tertiary-care hospital in western Maharashtra, India. Singapore Med J. 2012;53(4):264-7.

17. Laspina F, Samudio M, Cibils D, Fariña N, et al. Epidemiological characteristics of microbiological results on patients with infectious corneal ulcers: A 13-year survey in Paraguay. Graefe's Arch Clin Exp Ophthalmol 2004;242(3):204-9.

18. Norina T, Raihan S, Bakiah S, Ezanee M, Liza-Sharmini A, Wan Hazzaba W. Microbial keratitis: Aetiological diagnosis and clinical features in patients admitted to Hospital Universiti Sains Malaysia Singapore Med J 2008; 49 (1): 68

19. Al-Ghafri A, Al-Raisi A. The epidemiology of nonviral microbial keratitis in a tertiary care center in Muscat, Oman. Oman J Ophthalmol. 2018;11(3):213.

20. Arrúa M, Laspina $F$, Samudio M, Fariña N, Cibils D, Sanabria R, et al. Queratitis infecciosas. Características clínicas y microbiológicas . Período 2003-2006. Mem. Inst. Investig. Cienc. Salud 2008;6(1):514.

21. Singh A, Singh D, Kumar $S$, Mishra A, Verma $R$, Mishra $V$. A retrospective study of fungal corneal ulcer from the western part of Uttar Pradesh. Int J Res Med Sci. 2015;3(4):880.

22. Estopinal C, Ewald M. Geographic Disparities in the Etiology of Bacterial and Fungal Keratitis in the United States of America. Semin Ophthalmol. 2016;31(4):345-52.

23. Farias R, Pinho L, Santos R. Epidemiological profile of infectious keratitis. Rev Bras Oftalmol. 2017;76(3):116-20.

24. Wozniak RA, Aquavella J V. Antibiotics in ophthalmology practice. Expert Rev Ophthalmol [Internet]. Taylor \& Francis; 2017;12(3):243-50. 